\title{
Immunohistochemical demonstration of neurone specific enolase in bone marrow infiltrated by
} neuroblastoma

\author{
J M Caillaud, F Martinez-Madrigal, O Hartmann, C Carlu
}

\begin{abstract}
One hundred and two bone marrow samples were analysed by histological and immunohistochemical methods for neurone specific enolase (NSE). The biopsies were performed to determine the extent of bone marrow disease in 84 neuroblastomas, nine embryonal rhabdomyosarcomas, five Ewing's sarcomas, two cases of Hodgkin's disease and two lymphoblastic lymphomas. Twenty seven $(32 \%)$ of neuroblastoma bone marrows showed metastases by conventional histological techniques and 33 $(39 \%)$ after immunohistochemical staining with NSE. Five embryonal rhabdomyosarcomas, five Ewing's sarcomas, and two lymphoblastic lymphomas showed bone marrow metastases. Only one of these cases was reactive for NSE. NSE represents a very sensitive immunomarker for the follow up of neuroblastoma and improves detection of bone marrow invasion by neuroblastoma.
\end{abstract}

Staging of neuroblastoma includes examination of bone marrow trephine biopsy specimens for detection of bone marrow infiltration at diagnosis and residual tumour after chemotherapy. In both cases histological diagnosis of metastatic neuroblastoma in bone marrow may be difficult. In micrometastasis malignant cells are intermingled with haematopoietic cells following the architectural pattern of bone marrow. Under these conditions histological diagnosis is difficult or even impossible. After chemotherapy metastatic foci become fibrotic, making the detection of residual neoplastic cells difficult. To avoid these difficulties, we performed immunostaining for neurone specific enolase (NSE) in 102 bone marrows previously studied by conventional histological techniques. NSE is a very sensitive marker for neuroblastoma, ${ }^{1}$ but it is also found in other poorly differentiated childhood tumours. ${ }^{2}$

\section{Methods}

Bone marrow trephine biopsy specimens from 102 patients were analysed. Initial diagnosis of primary tumour had been made according to clinical, radiological, biochemical and histopathological findings. There were 84 neuroblastomas, nine embryonal rhabdomyosarcomas, five Ewing's sarcomas; two cases of Hodgkin's disease and two lymphoblastic lymphomas.

All specimens were fixed in Bouin's solution and later decalcified in EDTA medium before fixing in paraffin wax. For each case $6 \mu \mathrm{m}$ sections were stained with haematoxylin and eosin and safranin (HES) and Gordon and Sweet's stain for reticulin. Immunohistochemical localisation of NSE was studied by the peroxidase-antiperoxidase method. ${ }^{3}$

The sections were incubated with rabbit polyclonal antisera directed against NSE (Dakopatts, Denmark) at a dilution of 1 in 50. The peroxidase-antiperoxidase complex was used in combination with diaminobenzidine tetrahydrochloride (Polysciences, Warrington). Endogenous peroxidase was inhibited with hydrogen peroxide $\left(\mathrm{H}_{2} \mathrm{O}_{2}\right)$.

Non-immunised rabbit serum was used as negative control.

\section{Results}

HISTOPATHOLOGICAL FINDINGS

Twenty seven of the 84 patients with neuroblastoma had metastases (fig 1) (table) by HES. Fifty four bone marrows were free of metastasis and three others showed residual fibrosis but without obvious neoplastic cells ("cicatricial type"). Twelve bone marrow specimens from patients with tumours other than neuroblastoma showed metastatic cellsfive embryonal rhabdomyosarcomas, five Ewing's sarcomas, and two lymphoblastic lymphomas (table).

\section{IMMUNOHISTOCHEMICAL FINDINGS}

Immunohistochemical results are summarised in the table. Thirty three bone marrow specimens were positive for NSE. In 49 cases immunoreactivity for NSE was negative; two cases were difficult to interpret. Only one of the 18 bone marrow specimens of patients with tumours other than neuroblastoma was positive for the NSE. The positive case corresponded to Ewing's sarcoma. Positivity for NSE in bone marrow invaded by neuroblastoma was observed in both poorly differentiated cells (fig 2) and in mature ganglion cells (fig 3 ). The reactivity was intracytoplasmic and in the fibrillary processes. The reaction was particularly strong in metastatic bone marrow previously identified by HES. No metastatic bone marrow previously identified by HES were negative for NSE. Five bone marrow specimens that were considered to be nonmetastatic by HES exhibited small groups of neoplastic cells positive for NSE (fig 3). Only one of the three "cicatricial type" bone 
Figure 1 Bone marrow biopsy specimen showing typical metastasis of neuroblastoma. Note the fibrillary aspect of the malignant stroma

(haematoxylin and eosin).

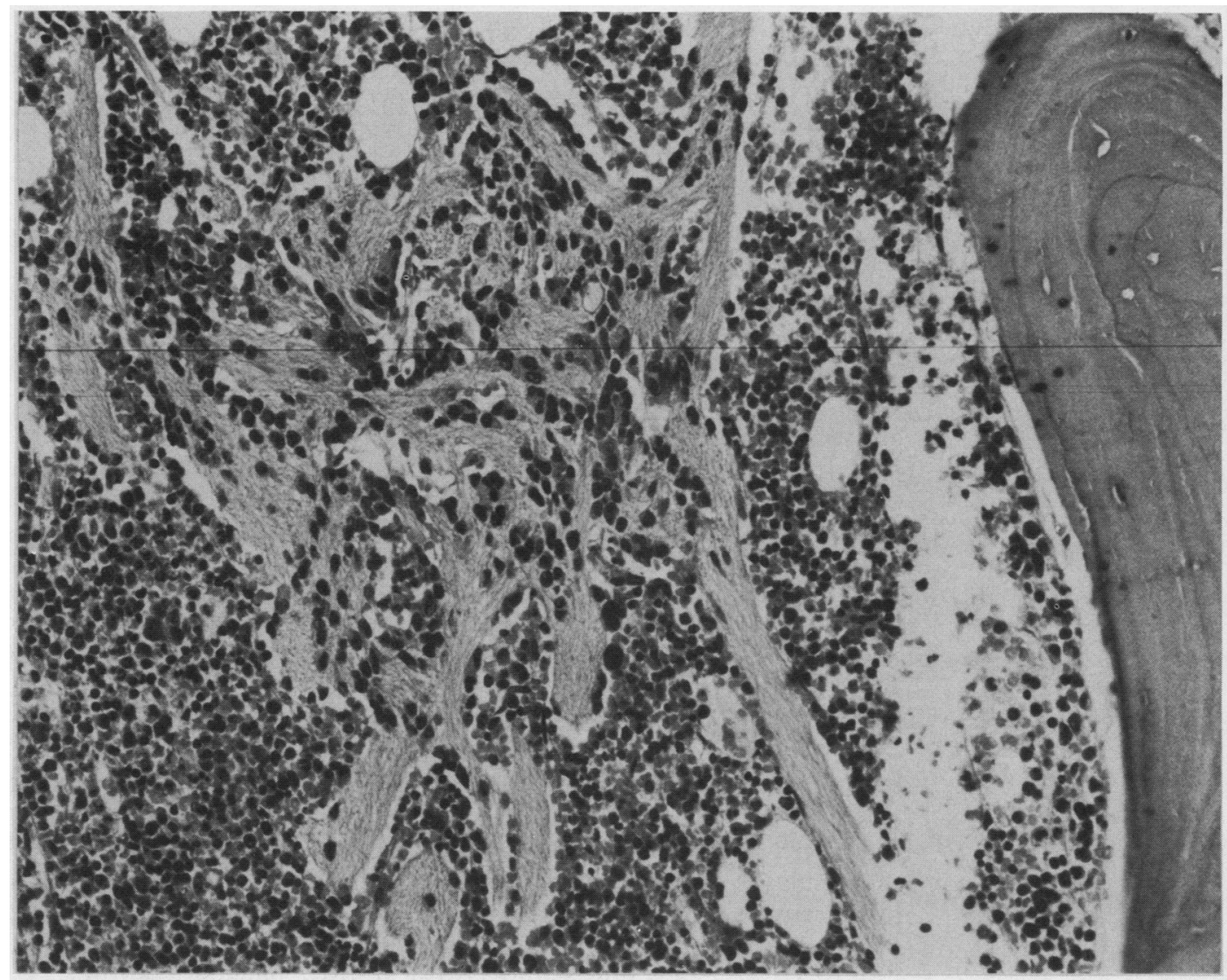

Histology and immunohistochemistry

\begin{tabular}{lllll}
\hline Histology and immunohistochemistry & Metastatic & Non-metastatic & "Cicatricial” & Total \\
\hline Neuroblastoma & 27 & 54 & 3 & 84 \\
NSE positive & 27 & 5 & 1 & 33 \\
Other tumours & 12 & 6 & - & 18 \\
NSE positive & 1 & - & - & 1 \\
\hline
\end{tabular}

marrows showed NSE positive cells intermingled with the fibrotic tissue. The two cases interpreted as doubtful for NSE consisted of two non-metastatic bone marrows by conventional histological techniques. These cases showed structures positive for NSE but without visible nuclei. We eventually considered
Figure 2

Immunostaining for NSE in a metastatic bone marrow neuroblastoma. Note strong reactivity.

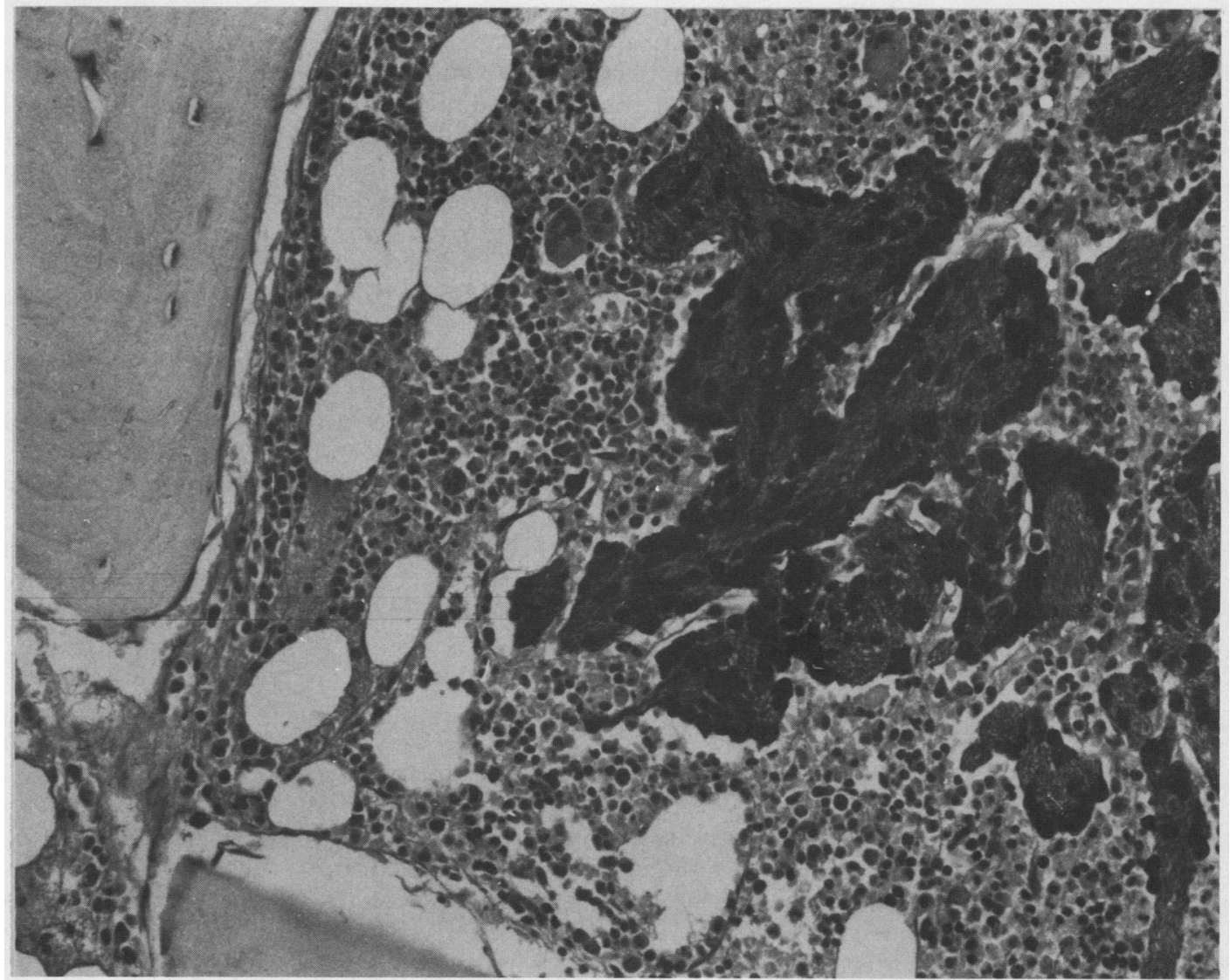


Figure 3 Isolated neuroblastoma cell in a bone marrow biopsy specimen. Immunostaining for NSE permits recognition of the cell intermingled with the haematopoietic cells. These malignant cells correspond to mature ganglion cells.

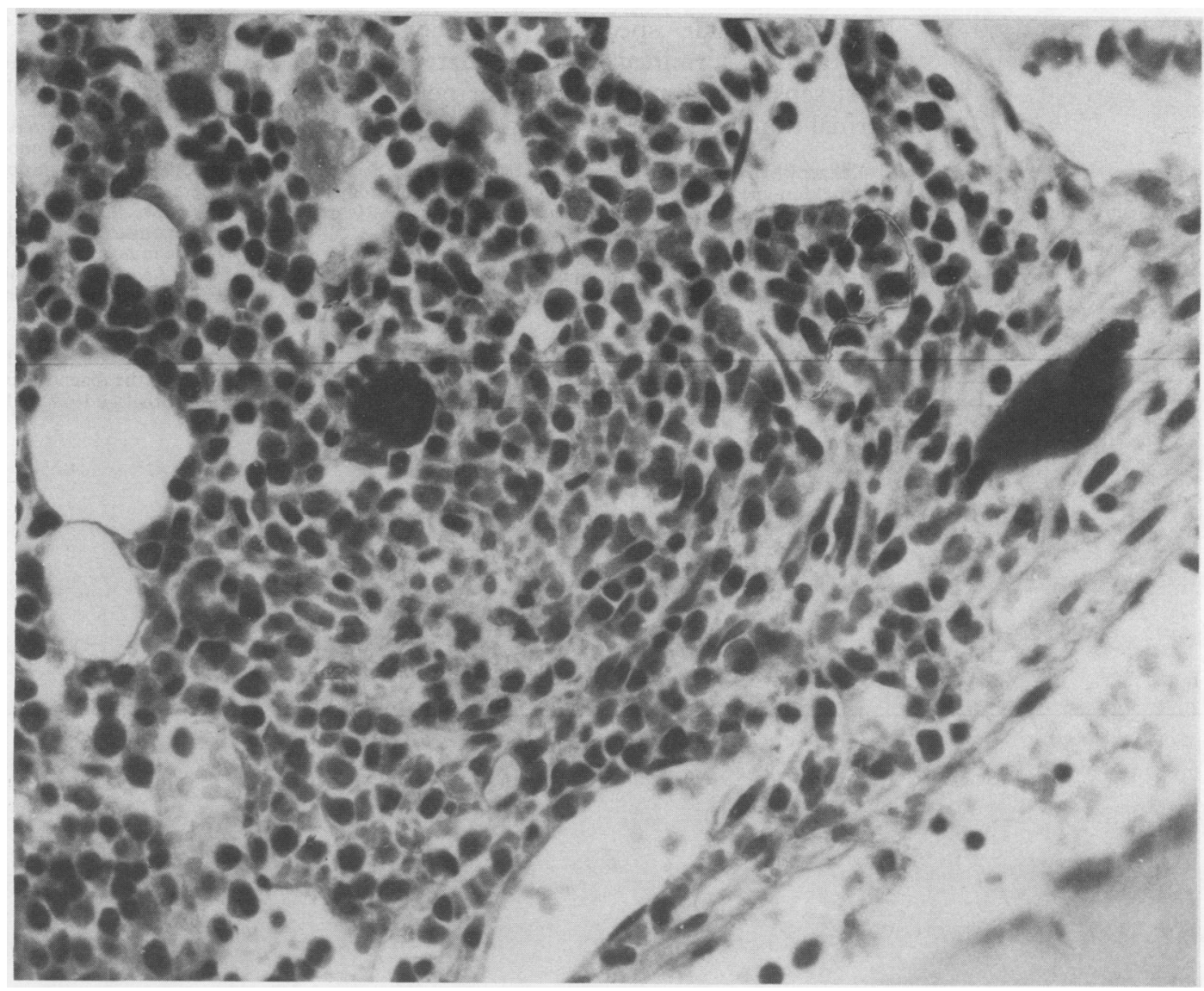

these cases to be non-metastatic. No immunoreactivity was found in granulocytic cells or osteoblastic cells in any bone marrows.

\section{Discussion}

This study shows that there is consistent strong positivity for NSE in bone marrow invaded by neuroblastoma detected by conventional histological techniques. Nevertheless, some poorly differentiated neuroblastoma or inappropriately fixed specimens do not manifest NSE reactivity. ${ }^{4}$ NSE positive cells correspond to immature neuroblastic cells or mature ganglion cells. No reactivity was found in haematopoietic cells.

In this series of 84 cases of neuroblastoma immunoreactivity for NSE in bone marrow specimens permitted the diagnosis of metastases in $39 \%$ of cases; metastases were found in $32 \%$ by conventional histological techniques. Therefore immunostaining represents a more sensitive method of detecting micrometastases in bone marrow.

Two of our cases were difficult to interpret because of the presence of acellular positive structures. In these two cases we failed to find nuclei in several sections. These cases were not metastatic according to conventional histology. Such structures could have been interpreted as residual tumour cells, as similar formations have been found in bone marrows with typical metastases.

The three so-called "cicatricial-type" were cases studied after chemotherapy. These showed fibrotic foci probably corresponding to regressing metastases, but no neoplastic cells were shown by conventional histology. Immunostaining for NSE detected neoplastic cells in one of these bone marrows and so showed the persistence of tumour.

Immunostaining by the peroxidase antiperoxidase technique in bone marrow specimens requires the inhibition of endogenous peroxidase that is normally present in some granulocytic cells. This is easily accomplished with $\mathrm{H}_{2} \mathrm{O}_{2}$. Otherwise metastases may be falsely detected. Nevertheless, this might represent a problem of interpretation, as none of our bone marrow specimens showed reactivity in granulocytic cells or osteoblasts. False negativity after decalcification of tissues has been considered a limitation of immunoperoxidase methods. When immunoreactivity was compared in decalcified and non-decalcified tissues, however, there was no considerable difference in immunoreactivity. ${ }^{5}$ Only one of the bone marrows invaded by other tumours was positive for NSE. The case corresponded to Ewing's sarcoma of the tibia. The presence of NSE in some cases of Ewing's sarcoma is consistent with the findings of recent studies. ${ }^{2}$

We conclude that immunostaining for NSE increases the rate of detection of bone marrow invasion by neuroblastoma cells. It detects very small aggregates of malignant cells. The prognostic value of these small metastases and particularly the mature ganglion cells (fig 3), however, remains questionable.

Many other antibodies have been tested for diagnosis of neuroblastoma, ${ }^{6}$ but most of them are only useful in frozen sections or in cytology. 
Moreover, their specificity has not been accurately determined. NSE is currently the most sensitive immunomarker for neuroectodermal tumours. ${ }^{7-9}$

1 Zeltzer PM, Marangos PS, Evans AE, Schenider SL. Serum neuron-specific enolase in children with neuroblastoma. Relationship to stage and disease course. Cancer 1986; 57:1230-4.

2 Tsokos M, Linnoila RI, Chandra RS, Triche TJ. Neuron specific enolase in the diagnosis of neuroblastomg and other small round-cell tumors in children. Hum Pathol 1984;15:575-84.

3 Sternberger L. Immunohistochemistry. 2nd ed. New York John Wiley, 1979.

4 Seshi B, True L, Carter D, Rosai J. Immunohistochemical characterization of a set of monoclonal antibodies to human neuron specific enolase. Am J Pathol 1988;131: 258-69.

5 Mukai $\mathrm{K}$, Yoshimura S, Anzai M. Effects of decalcification (19) J Surg Pathol 1986; 10:413-9.

6 Kemshead JT, Goldman A, Fritshy J, Malpas JS, Pritchard $J$. Use of panels of monoclonal antibodies in the differential diagnosis of neuroblastoma and lymphoblastic disorders. Lancet 1983; i:12-5.

7 Vinores SA, Bonnin JM, Rubinstein LJ, Marangos PJ. Immunohistochemical demonstration of neuron-specific enolase in neoplasms of the CNS and other tissues. Arch Pathol Lab Med 1984;108:536-40.

8 Triche TJ, Askin FB. Neuroblastoma and the differential diagnosis of small-round-blue-cell tumors. Hum Pathol 1983;14:569-95.

9 Dhillon AP, Rode J, Leathem A. Neuron-specific enolase: an aid to the diagnosis of melanoma and neuroblastoma. Histopathology 1982;6:81-92.

\section{Eponyms in pathology...}

SHIGA, Kyoshi (1870-1957) was a Japanese microbiologist who was born in Sendai, northern Japan. He graduated from Tokyo university in 1896. After two years as Erlich's assistant, from 1901-1903, he returned to Japan to take charge of a department at the Kitasito institute for infectious diseases in 1904. Sixteen years later he was appointed professor and dean of the medical faculty of the Keyo imperial university. In 1898 he reported the discovery of the organism causing bacillary dysentery. The genus, Shigella, now carries his name. 